\title{
Association Between Red Blood Cell Distribution Width and Intraoperative Transfusion in Patients Undergoing Living Donor Liver Transplantation: a Retrospective Single-center Cohort Study
}

\author{
Ji Hoon Sim \\ Asan Medical Center \\ Hye-Mee Kwon \\ Asan Medical Center \\ In-Gu Jun \\ Asan Medical Center \\ Sung-Hoon Kim \\ Asan Medical Center
}

Bomi Kim

Asan Medical Center

Sehee Kim

Asan Medical Center

Jun-Gol Song ( $\sim$ jungol.song@amc.seoul.kr)

Asan Medical Center, University of Ulsan College of Medicine https://orcid.org/0000-0002-6076-6978

Gyu-Sam Hwang

Asan Medical Center

\section{Research}

Keywords: bleeding, liver transplantation, living donor, red blood cell distribution width, survival, transfusion

Posted Date: April 28th, 2021

DOI: https://doi.org/10.21203/rs.3.rs-463926/v1

License: (c) (1) This work is licensed under a Creative Commons Attribution 4.0 International License.

Read Full License 


\section{Abstract}

Background: Living donor liver transplantation (LDLT) has been associated with massive bleeding and increased blood transfusion requirements. However, information on indicators predicting bleeding and transfusion in LDLT is limited. Recent studies have reported a correlation between red cell distribution width (RDW) and bleeding risk. This study investigates the association between RDW and intraoperative blood transfusion in patients receiving LDLT.

Methods: This retrospective study analyzed 2548 patients who underwent LDLT between January 2010 and October 2019. The patients were divided into four groups based on preoperative RDW quartiles: Q1 (<13.9), Q2 (13.9-15.2), Q3 (15.2-17.4), and Q4 ( $\geq 17.4)$, and transfusion requirement and surgical outcomes were assessed. The risk factors for intraoperative transfusion were evaluated by multivariate regression analysis. The predictive power of RDW was assessed through receiver operating characteristic (ROC) and integrated discrimination improvement (IDI) analysis.

Results: There were significant differences in incidence of intraoperative transfusion according to preoperative RDW quartile (Q1 vs Q2 vs Q3 vs Q4: $47.3 \%$ vs. $78.1 \%$ vs. $91.8 \%$ vs. $96.2 \%, P<0.001$ ). Q4 had poor surgical outcomes, such as acute kidney injury (adjusted odds ratio [OR]: 1.91, 95\% Cl: 1.44-2.54, $P<0.001)$. In the multivariate logistic analysis, RDW, age, sex, diabetes mellitus, coronary artery disease, model for end-stage liver disease scores, and total ischemic time were risk factors for transfusion. In ROC and IDI analysis, RDW had predictive power for intraoperative transfusion ( $P=0.023$ in IDI).

Conclusions: Preoperative RDW is a potential predictor of intraoperative transfusion and postoperative acute kidney injury in patients receiving LDLT.

\section{Introduction}

Liver transplantation (LT) is one of the most complex surgeries for patients with end-stage liver disease and represents a great challenge for surgeons and anesthesiologists.[1] Historically, LT has been associated with massive intraoperative bleeding and large transfusions.[2] Although recent advances in surgical techniques, anesthetic skills, and perioperative management have reduced intraoperative bleeding, $[3,4]$ LT is still associated with high bleeding risk and increased blood transfusion requirements. [5] Despite numerous efforts to find predictors of intraoperative bleeding and transfusion in LT, only limited information is available to date.[6, 7]

The red blood cell distribution width (RDW) is a measure of the variation in the size of red blood cells in a blood sample and part of a standard complete blood count.[8] RDW is a simple and objective indicator of patient survival and complications even in acute and chronic diseases such as heart failure, stroke, and various cancers.[9-13] Higher RDW indicates more than expected variations in red blood cell size. Several recent studies have reported that RDW is correlated with bleeding risk and transfusion for some diseases and procedures.[14-16] 
However, there are few studies on the association between preoperative RDW and intraoperative transfusion in LT. Therefore, in this study, we evaluated the association between preoperative RDW and intraoperative blood transfusion in patients who underwent living donor liver transplantation (LDLT).

\section{Methods}

\section{Study design and patient population}

The institutional review board of the Asan Medical Center (Protocol No. 2021 - 0243) approved this retrospective study, and because of the retrospective nature of our study, the requirement for written informed consent was waived. We analyzed all the patients who underwent LDLT for end-stage liver disease between January 2010 and October 2019. The following patients were excluded: patients aged < 18 or $\geq 80$ years; patients with hematologic disorders; patients taking anticoagulants, such as aspirin, warfarin, or antiplatelet agents; patients undergoing emergency surgery; and patients with incomplete or missing data.

\section{Anesthetic technique}

Hemodynamic monitoring and general anesthesia were performed according to our institutional standards.[17] Briefly, anesthesia was maintained using desflurane or sevoflurane, a mixture of $50 \% \mathrm{O}_{2}$ and $50 \%$ air, and continuous intravenous infusion of fentanyl. Invasive arterial-venous pressure monitoring was performed by radial and femoral arterial catheterization. Central venous pressure monitoring was routinely performed, and a pulmonary arterial catheter was inserted to monitor hemodynamic variables via a Vigilance monitor (Vigilance II, Edwards Lifesciences LLC). During anesthesia, fluids or vasopressors were administered by an anesthesiologist based on the patient's mean blood pressure (MBP) and hemodynamics. In cases of low systemic vascular resistance, MBP was maintained by the continuous infusion of inotropic agents, such as norepinephrine, vasopressin, or terlipressin. Plasma and $5 \%$ or $20 \%$ albumin were administered during anesthesia. If the plasma hemoglobin level was $<8 \mathrm{~g} / \mathrm{dL}$ or massive bleeding was expected due to intraoperative bleeding, packed red blood cells (PRBCs) were transfused, and hemoglobin level was maintained at $>10 \mathrm{~g} / \mathrm{dL}$ in patients with ischemic heart disease.

\section{Data collection and outcome assessment}

Patient characteristics and perioperative variables were collected using the medical record system of our institution. Patient characteristics included age, sex, body mass index (BMI), diabetes mellitus (DM), hypertension (HTN), coronary artery disease (CAD), and model for end-stage liver disease (MELD) scores. Variables related to patients' tumor etiology included hepatitis B virus (HBV), hepatitis C virus (HCV), alcoholic liver cirrhosis, and combined hepatocellular carcinoma (HCC). Donor-related variables included age, sex, and BMI.

Preoperative laboratory values included levels of white blood cells (WBCs), hemoglobin, platelet, fibrinogen, albumin, aspartate transaminase (AST), alanine transaminase (ALT), total bilirubin, sodium, 
serum creatinine ( $\mathrm{sCr}$ ), international normalized ratio (INR), and estimated glomerular filtration rate (eGFR). sCr levels were checked daily from postoperative day 1 to day 7 to confirm acute kidney injury (AKI). Data on preoperative neutrophil-lymphocyte ratio (NLR), platelet-lymphocyte ratio (PLR), prognostic nutritional index (PNI), C-reactive protein/albumin ratio, and RDW were also collected. NLR was defined as the ratio of absolute neutrophil count to absolute lymphocyte count, and PLR was defined as the ratio of absolute platelet count to absolute lymphocyte count, respectively. PNI was calculated using serum albumin level and total lymphocyte count.[18]

Intraoperative variables included operation time, total ischemic time, post-reperfusion syndrome, total volume of fluid administered, and urine output. Data on intraoperative and postoperative transfusion, massive transfusion, hospital stay, postoperative AKI, intensive care unit (ICU) stay, 1-year graft failure, 1year survival, and overall survival were collected. Massive transfusion was defined as $\geq 10$ units of PRBCs within 24 hours, $\geq 4$ units of PRBCs within 1 hour, or replacement of $>50 \%$ of the total blood volume by blood products within 3 hours.[19] Total blood volume for adults was based on Gilcher's rule of five for blood volume.[19]

\section{Primary and secondary endpoints}

The primary endpoints were to compare intraoperative transfusion according to quartiles of preoperative RDW. The secondary endpoints were to analyzed risk factors associated with intraoperative transfusion and evaluate surgical outcomes, such as postoperative AKI. Postoperative AKI was defined by the Kidney Disease Improving Global Outcomes classification: $\mathrm{sCr}$ level increased by at least 1.5 times of baseline value within 7 days after surgery or an $\mathrm{sCr}$ level increase of $0.3 \mathrm{mg} / \mathrm{dL}$ within 48 hours after surgery.[20] In addition, we evaluated the predictive power of RDW for intraoperative blood transfusion through receiver operating characteristic (ROC) and integrated discrimination improvement (IDI) analyses.

\section{Statistical analysis}

Data are described as means \pm standard deviations, medians (interquartile ranges), or numbers (proportions), as appropriate. The study variables were compared between four groups created according to quartiles of preoperative RDW. We used a chi-squared test or Fisher's exact test for categorical variables and Student's t-test or Mann-Whitney U-test for continuous variables. Multiple logistic regression analysis was applied to identify the risk factors for intraoperative transfusion. All variables with $P<0.1$ in the univariate analysis were included in the multivariate analysis. The predictive value of RDW for intraoperative transfusion was evaluated using ROC and IDI analyses. A $P<0.05$ was considered statistically significant. All data were analyzed using SPSS Statistics version 22.0 for Windows (IBM Corp., Armonk, NY) and R version 3.1.2 (R Foundation for Statistical Computing, Vienna, Austria).

\section{Results}

Of 2658 patients who underwent LDLT, 110 patients were excluded according to the exclusion criteria. In total, 2,548 patients were included and divided into four groups by RDW quartile: Q1 $(<13.9, n=609)$, Q2 $(13.9-15.2, n=626), Q 3(15.2-17.4, n=661), Q 4(\geq 17.4, n=652)$. The characteristic, etiological, donor- 
related, laboratory, and intraoperative variables of the patients are shown in Table 1 . The groups in higher quartiles were younger $(P=0.001)$, more likely to be female $(P<0.001)$, more likely to have a history of $\operatorname{DM}(P=0.007)$, and less likely to have a history of HTN $(P<0.001)$. Moreover, these groups had higher MELD scores $(P<0.001)$, a higher incidence of alcoholic liver cirrhosis, lower BMI $(P=0.003)$, and a lower incidence of HBV, HCV, and HCC. There was no significant association between preoperative RDW and donor-related variables, such as age $(P=0.621)$, sex $(P=0.755)$, and BMI $(P=0.773)$ (Table 1$)$. 
Table 1

Baseline characteristics, pre- and intraoperative variables, and surgical outcomes of the study population

\section{Red blood cell distribution width}

\begin{tabular}{lllll} 
Quartile $\otimes$ & Quartile $\otimes$ & Quartile $\otimes$ & Quartile $\otimes$ & $\begin{array}{l}P \text { - } \\
\text { value }\end{array}$ \\
$\leq<13.9$ & $13.9-15.2$ & $15.2-17.4$ & $\geq 17.4$ & \\
\cline { 1 - 2 }$(n=609)$ & $(n=626)$ & $(n=661)$ & $(n=652)$ &
\end{tabular}

\section{Demographic variables}

\begin{tabular}{llllll} 
Age, year & $53.97 \pm 7.62$ & $54.21 \pm 8.19$ & $53.70 \pm 7.87$ & $52.48 \pm 9.05$ & 0.001 \\
\hline Male & $510(83.74)$ & $477(76.20)$ & $480(72.62)$ & $417(63.96)$ & $<$ \\
\hline BMl, kg m${ }^{-2}$ & $24.52 \pm 3.01$ & $24.43 \pm 3.17$ & $24.11 \pm 3.55$ & $23.87 \pm 3.91$ & 0.003 \\
\hline DM & $134(22.00)$ & $174(27.80)$ & $189(28.59)$ & $147(22.55)$ & 0.007 \\
\hline HTN & $163(26.77)$ & $104(16.61)$ & $105(15.89)$ & $88(13.50)$ & $<$ \\
\hline CAD & $52(8.54)$ & $55(8.79)$ & $56(8.47)$ & $45(6.9)$ & 0.593 \\
\hline MELD scores & $9.32 \pm 3.69$ & $12.71 \pm 5.43$ & $15.62 \pm 6.81$ & $21.91 \pm 9.89$ & $<$ \\
\hline Etiology & & & & & 0.001 \\
\hline HBV & $472(77.50)$ & $402(64.22)$ & $367(55.52)$ & $301(46.17)$ & $<$ \\
\hline HCV & $45(7.39)$ & $50(7.99)$ & $60(9.08)$ & $32(4.91)$ & 0.001 \\
\hline Alcoholic liver cirrhosis & $58(9.52)$ & $108(17.25)$ & $151(22.84)$ & $200(30.67)$ & $<$ \\
\hline HCC & $496(81.44)$ & $343(54.79)$ & $257(38.88)$ & $175(26.84)$ & $<$ \\
\hline
\end{tabular}

\section{Donor-related variables}

Study groups according to RDW quartiles: Q1 (< 13.9), Q2 (13.9-15.2), Q3 (15.2-17.4), Q4 (>17.4).

BMI, body mass index; DM, diabetes mellitus; HTN, hypertension; CAD, coronary artery disease; MELD, model for end-stage liver disease; HBV, hepatitis B virus; $\mathrm{HCV}$, hepatitis $\mathrm{C}$ virus; $\mathrm{HCC}$, hepatocellular carcinoma; WBC, white blood cell; INR, international normalized ratio; AST, aspartate aminotransferase; ALT, alanine aminotransferase; eGFR, estimated glomerular filtration rate; NLR, neutrophil to lymphocyte ratio; PLR, platelet to lymphocyte ratio; PNI, prognostic nutritional index; CRP, C-reactive protein; RDW, red cell distribution width; AKI, acute kidney injury; ICU, intensive care unit; SD, standard deviation.

Values are expressed as means \pm standard deviations, medians (interquartile ranges), or absolute numbers (percentages). 
Red blood cell distribution width

\begin{tabular}{|c|c|c|c|c|c|}
\hline Age & $27.93 \pm 8.10$ & $27.78 \pm 7.71$ & $27.64 \pm 7.65$ & $28.21 \pm 7.99$ & 0.621 \\
\hline Male & 438 (71.92) & $434(69.33$ & $471(71.26)$ & $466(71.47)$ & 0.755 \\
\hline BMI & $23.13 \pm 3.04$ & $23.01 \pm 2.81$ & $23.09 \pm 2.96$ & $22.99 \pm 3.11$ & 0.773 \\
\hline \multicolumn{6}{|l|}{ Laboratory variables } \\
\hline WBC count & $3.81 \pm 1.71$ & $3.26 \pm 1.72$ & $3.68 \pm 2.75$ & $4.68 \pm 3.55$ & $\begin{array}{l}< \\
0.001\end{array}$ \\
\hline Hemoglobin level & $12.96 \pm 1.79$ & $11.37 \pm 1.87$ & $10.03 \pm 1.71$ & $9.04 \pm 1.51$ & $\begin{array}{l}< \\
0.001\end{array}$ \\
\hline Platelet count & $96.54 \pm 48.14$ & $68.80 \pm 47.90$ & $66.78 \pm 46.79$ & $70.44 \pm 52.87$ & $\begin{array}{l}<.001 \\
0.001\end{array}$ \\
\hline INR & $1.17 \pm 0.23$ & $1.38 \pm 0.38$ & $1.51 \pm 0.41$ & $1.87 \pm 0.86$ & $\begin{array}{l}< \\
0.001\end{array}$ \\
\hline Fibrinogen level & $\begin{array}{l}219.47 \pm \\
67.79\end{array}$ & $\begin{array}{l}182.44 \pm \\
69.03\end{array}$ & $\begin{array}{l}171.36 \pm \\
75.82\end{array}$ & $\begin{array}{l}140.82 \pm \\
70.38\end{array}$ & $\begin{array}{l}<.001 \\
0.001\end{array}$ \\
\hline Albumin level & $3.47 \pm 0.48$ & $3.05 \pm 0.53$ & $2.97 \pm 0.58$ & $3.06 \pm 0.57$ & $\begin{array}{l}< \\
0.001\end{array}$ \\
\hline AST level & $\begin{array}{l}49.24 \pm \\
252.82\end{array}$ & $\begin{array}{l}63.00 \pm \\
348.12\end{array}$ & $50.39 \pm 41.80$ & $74.32 \pm 81.77$ & 0.145 \\
\hline ALT level & $\begin{array}{l}40.75 \pm \\
233.47\end{array}$ & $\begin{array}{l}40.26 \pm \\
213.10\end{array}$ & $29.08 \pm 31.66$ & $42.34 \pm 62.97$ & 0.439 \\
\hline Total bilirubin level & $1.07 \pm 1.03$ & $2.00 \pm 2.10$ & $4.13 \pm 6.14$ & $11.77 \pm 12.37$ & $\dot{0.001}$ \\
\hline Sodium level & $140.01 \pm 3.19$ & $138.78 \pm 4.35$ & $137.29 \pm 5.37$ & $136.31 \pm 5.71$ & $\begin{array}{l}< \\
0.001\end{array}$ \\
\hline Creatinine level & $0.89 \pm 0.73$ & $0.89 \pm 0.78$ & $0.93 \pm 0.84$ & $1.05 \pm 0.99$ & 0.007 \\
\hline $\begin{array}{l}\text { eGFR, } \mathrm{mL} / \mathrm{min} / 1.73 \\
\mathrm{~m}^{2}\end{array}$ & $76.08 \pm 16.04$ & $73.78 \pm 18.22$ & $71.26 \pm 18.86$ & $69.02 \pm 21.48$ & $\begin{array}{l}< \\
0.001\end{array}$ \\
\hline
\end{tabular}

Study groups according to RDW quartiles: Q1 (<13.9), Q2 (13.9-15.2), Q3 (15.2-17.4), Q4 (>17.4).

BMI, body mass index; DM, diabetes mellitus; HTN, hypertension; CAD, coronary artery disease; MELD, model for end-stage liver disease; HBV, hepatitis B virus; $\mathrm{HCV}$, hepatitis $\mathrm{C}$ virus; $\mathrm{HCC}$, hepatocellular carcinoma; WBC, white blood cell; INR, international normalized ratio; AST, aspartate aminotransferase; ALT, alanine aminotransferase; eGFR, estimated glomerular filtration rate; NLR, neutrophil to lymphocyte ratio; PLR, platelet to lymphocyte ratio; PNI, prognostic nutritional index; CRP, C-reactive protein; RDW, red cell distribution width; AKI, acute kidney injury; ICU, intensive care unit; SD, standard deviation.

Values are expressed as means \pm standard deviations, medians (interquartile ranges), or absolute numbers (percentages). 


\section{Red blood cell distribution width}

$\begin{array}{llllll}\text { NLR } & 2.23 \pm 2.28 & 2.83 \pm 3.30 & 3.79 \pm 4.38 & 4.69 \pm 4.72 & < \\ \text { PLR } & 92.92 \pm 47.30 & 90.21 \pm 61.09 & 101.07 \pm & 101.39 \pm & 0.001 \\ \text { PNI } & 40.53 \pm 6.03 & 34.92 \pm 5.79 & 33.65 \pm 6.10 & 34.81 \pm 5.98 & < \\ \text { CRP/albumin } & 0.10 \pm 0.24 & 0.17 \pm 0.31 & 0.32 \pm 0.53 & 0.38 \pm 0.69 & < \\ & & & & & 0.001 \\ \text { RDW } & 13.04 \pm 0.55 & 14.49 \pm 0.37 & 16.10 \pm 0.64 & 20.33 \pm 2.69 & < \\ & & & & & 0.001\end{array}$

\section{Intraoperative variables}

\begin{tabular}{|c|c|c|c|c|c|}
\hline Operation time, min & $\begin{array}{l}781.83 \pm \\
109.01\end{array}$ & $\begin{array}{l}830.90 \pm \\
120.23\end{array}$ & $\begin{array}{l}840.77 \pm \\
118.47\end{array}$ & $\begin{array}{l}847.35 \pm \\
126.89\end{array}$ & $\begin{array}{l}< \\
0.001\end{array}$ \\
\hline $\begin{array}{l}\text { Total ischemic time, } \\
\text { min }\end{array}$ & $\begin{array}{l}123.42 \pm \\
39.30\end{array}$ & $\begin{array}{l}125.11 \pm \\
28.47\end{array}$ & $\begin{array}{l}127.71 \pm \\
28.65\end{array}$ & $\begin{array}{l}126.63 \pm \\
30.42\end{array}$ & 0.071 \\
\hline $\begin{array}{l}\text { Post-reperfusion } \\
\text { syndrome }\end{array}$ & $342(56.16)$ & $388(61.98)$ & $405(61.27)$ & $418(64.11)$ & 0.030 \\
\hline Total fluids, mL/kg & $68.79 \pm 10.42$ & $67.75 \pm 10.96$ & $66.03 \pm 10.97$ & $64.66 \pm 12.08$ & $<_{0.001}^{<}$ \\
\hline Urine output, $\mathrm{mL} / \mathrm{kg} / \mathrm{hr}$ & $2.63 \pm 1.36$ & $2.24 \pm 1.33$ & $1.93 \pm 1.18$ & $1.87 \pm 1.25$ & $\dot{0} .001$ \\
\hline \multicolumn{6}{|l|}{ Transfusion } \\
\hline Intraoperative & $288(47.29)$ & $489(78.12)$ & $607(91.83)$ & $627(96.17)$ & $\begin{array}{l}<.001 \\
0.00\end{array}$ \\
\hline Massive ( $\geq 10$ units) & $68(11.17)$ & $177(28.27)$ & $276(41.75)$ & $343(52.61)$ & $\hat{0}_{0.001}$ \\
\hline
\end{tabular}

Study groups according to RDW quartiles: Q1 (<13.9), Q2 (13.9-15.2), Q3 (15.2-17.4), Q4 (> 17.4).

BMI, body mass index; DM, diabetes mellitus; HTN, hypertension; CAD, coronary artery disease; MELD, model for end-stage liver disease; HBV, hepatitis B virus; $\mathrm{HCV}$, hepatitis $\mathrm{C}$ virus; $\mathrm{HCC}$, hepatocellular carcinoma; WBC, white blood cell; INR, international normalized ratio; AST, aspartate aminotransferase; ALT, alanine aminotransferase; eGFR, estimated glomerular filtration rate; NLR, neutrophil to lymphocyte ratio; PLR, platelet to lymphocyte ratio; PNI, prognostic nutritional index; CRP, C-reactive protein; RDW, red cell distribution width; AKI, acute kidney injury; ICU, intensive care unit; SD, standard deviation.

Values are expressed as means \pm standard deviations, medians (interquartile ranges), or absolute numbers (percentages). 


\section{Red blood cell distribution width}

Postoperative

$403(66.17)$

$542(86.58)$

$602(91.07)$

$626(96.01)$

$<$

0.001

\section{Surgical outcomes}

\begin{tabular}{llllll} 
Hospital stay, days & $22.10 \pm 12.43$ & $25.52 \pm 14.39$ & $31.26 \pm 34.94$ & $36.62 \pm 40.13$ & $<.001$ \\
\hline ICU stay, days & $3.22 \pm 3.03$ & $4.72 \pm 5.89$ & $5.46 \pm 11.12$ & $7.13 \pm 12.33$ & $<$ \\
& & & & & 0.001 \\
AKI & $297(48.77)$ & $400(63.90)$ & $441(66.72)$ & $398(61.04)$ & 0.001 \\
\hline 1-year graft failure & $25(4.11)$ & $30(4.79)$ & $33(4.99)$ & $52(7.98)$ & 0.012 \\
\hline 1-year survival & $22(3.61)$ & $27(4.31)$ & $26(3.93)$ & $45(6.90)$ & 0.021 \\
\hline Overall survival & $75(12.32)$ & $73(11.66)$ & $81(12.25)$ & $95(14.57)$ & 0.413
\end{tabular}

Study groups according to RDW quartiles: Q1 (<13.9), Q2 (13.9-15.2), Q3 (15.2-17.4), Q4 (>17.4).

BMI, body mass index; DM, diabetes mellitus; HTN, hypertension; CAD, coronary artery disease; MELD, model for end-stage liver disease; HBV, hepatitis B virus; $\mathrm{HCV}$, hepatitis $\mathrm{C}$ virus; $\mathrm{HCC}$, hepatocellular carcinoma; WBC, white blood cell; INR, international normalized ratio; AST, aspartate aminotransferase; ALT, alanine aminotransferase; eGFR, estimated glomerular filtration rate; NLR, neutrophil to lymphocyte ratio; PLR, platelet to lymphocyte ratio; PNI, prognostic nutritional index; CRP, C-reactive protein; RDW, red cell distribution width; AKI, acute kidney injury; ICU, intensive care unit; SD, standard deviation.

Values are expressed as means \pm standard deviations, medians (interquartile ranges), or absolute numbers (percentages).

With regard to laboratory variables, the higher RDW quartile groups had higher WBC counts $(P<0.001)$, an increase in INR $(P<0.001)$, total bilirubin $(P<0.001)$ and sCr levels $(P=0.007)$, NLR $(P<0.001)$, PLR $(P=$ 0.009), and CRP/albumin ratio $(P<0.001)$, and a decrease in levels of hemoglobin $(P<0.001)$, platelets $(P<0.001)$, fibrinogen $(P<0.001)$, albumin $(P<0.001)$ and sodium $(P<0.001)$, eGFR $(P<0.001)$, and PNI $(P<0.001)$. There was no significant association between AST $(P=0.145)$ and ALT levels $(P=0.439)$, and preoperative RDW (Table 1). Furthermore, the groups with higher RDW had a significantly longer operation time $(P<0.001)$, a higher incidence of post-reperfusion syndrome, a lower volume of fluid administered, and lower urine output $(P<0.001)$. However, there were no significant intergroup differences in total ischemic time (Table 1).

\section{Primary endpoints}

The incidence of intraoperative transfusion according to RDW quartile is shown in Table 1. Of 2548 patients, 2011 patients (78.9\%) received intraoperative blood transfusions and 865 patients (33.9\%) 
received massive transfusions. The percentage of patients who required intraoperative transfusions in Q1, Q2, Q3, and Q4 was 47.3\% (288/609), 78.1\% (489/626), 91.8\% (607/661), and 96.2\% (627/652), respectively, with significant differences between these groups $(P<0.001)$. The percentage of patients requiring massive transfusions in these groups was $11.2 \%$ (68/609), 28.3\% (177/626), 41.8\% (276/661), and 52.6\% (343/652), respectively, with significant intergroup differences $(P<0.001)$, and the percentage of patients requiring postoperative transfusions in these quartile groups was $66.2 \%$ (403/609), $86.6 \%$ (542/626), 91.1\% (602/661), and 96.0\% (626/652), respectively, with significant differences between the groups $(P<0.001)$.

\section{Secondary endpoints}

The groups with higher RDW quartiles had prolonged hospital stay $(22.10,25.52,31.26$, and 36.62 days in Q1, Q2, Q3, and Q4, respectively; $P<0.001)$ and ICU stay $(3.22,4.72,5.46$, and 7.13 days in Q1, Q2, Q3, and Q4, respectively; $P<0.001)$, and a higher incidence of postoperative AKI $(48.8 \%, 63.9 \%, 66.7 \%$, and $61.0 \%$ in Q1, Q2, Q3, and Q4, respectively; $P<0.001$ ) (Table 1). Moreover, the patients with higher RDW quartiles had a higher rate of 1-year graft failure $(4.1 \%, 4.8 \%, 5.0 \%$, and $8.0 \%$ in Q1, Q2, Q3, and Q4, respectively; $P=0.012)$ and 1-year survival $(3.6 \%, 4.3 \%, 3.9 \%$, and $6.9 \%$ in Q1, Q2, Q3, and Q4, respectively; $P=0.021)$. However, there were no significant differences in overall survival $(12.3 \%, 11.7 \%, 12.3 \%$, and $14.6 \%$ in Q1, Q2, Q3, and Q4, respectively; $P=0.413$ ) (Table 1).

In the multivariate logistic analysis, preoperative RDW quartile was significantly associated with intraoperative transfusion (odds ratio [OR] 1.96, 95\% Cl 1.47-2.61, $P<0.001$ in Q2; OR 4.20, 95\% Cl 2.90$6.07, P<0.001$ in Q3; OR 4.99, 95\% Cl 3.08-8.09, $P<0.001$ in Q4) (Table 2). Additionally, age (OR 1.04, $95 \% \mathrm{Cl} 1.02-1.06, P<0.001$ ), male sex (OR 0.30, 95\% Cl 0.21-0.42, $P<0.001)$, DM (OR 1.77, 95\% Cl 1.32-2.38, $P<0.001$ ), CAD (OR 1.75, 95\% Cl 1.09-2.81, $P=0.019$ ), MELD scores (OR 1.33, 95\% Cl 1.27$1.39, P<0.001)$, and total ischemic time (OR 1.81, 95\% $\mathrm{Cl} 1.40-2.33, P<0.001)$ were significantly associated with intraoperative transfusion (Table 2). 
Table 2

Univariate and multivariable analysis of risk factors for intraoperative transfusion

\begin{tabular}{|c|c|c|c|c|c|c|}
\hline & \multicolumn{3}{|c|}{ Univariate } & \multicolumn{3}{|c|}{ Multivariable } \\
\hline & OR & $95 \% \mathrm{Cl}$ & $\begin{array}{l}P \text { - } \\
\text { value }\end{array}$ & OR & $95 \% \mathrm{Cl}$ & $\begin{array}{l}P \text { - } \\
\text { value }\end{array}$ \\
\hline RDW quartiles & & & $\begin{array}{l}<.001 \\
0\end{array}$ & & & $<0.001$ \\
\hline Q1 & $\begin{array}{l}1.00 \\
\text { (Ref.) }\end{array}$ & & & $\begin{array}{l}1.00 \\
\text { (Ref.) }\end{array}$ & & \\
\hline Q2 & 3.98 & $3.11-5.10$ & $<.001$ & 1.93 & $\begin{array}{l}1.45- \\
2.58\end{array}$ & $<0.001$ \\
\hline Q3 & 12.53 & $9.09-17.26$ & $\begin{array}{l}<.001 \\
0 .\end{array}$ & 4.04 & $\begin{array}{l}2.79- \\
5.84\end{array}$ & $<0.001$ \\
\hline Q4 & 27.95 & $\begin{array}{l}18.18- \\
42.98\end{array}$ & $<.001$ & 4.23 & $\begin{array}{l}2.86- \\
6.93\end{array}$ & $<0.001$ \\
\hline Age & 1.01 & $1.00-1.02$ & 0.107 & 1.04 & $\begin{array}{l}1.02- \\
1.06\end{array}$ & $<0.001$ \\
\hline Sex (male) & 0.29 & $0.22-0.39$ & $\begin{array}{l}<.001 \\
0.0\end{array}$ & 0.31 & $\begin{array}{l}0.22- \\
0.43\end{array}$ & $<0.001$ \\
\hline BMI & 0.97 & $0.94-0.99$ & 0.013 & 0.96 & $\begin{array}{l}0.93- \\
1.00\end{array}$ & 0.075 \\
\hline DM & 1.77 & $1.39-2.26$ & $<.001$ & 1.77 & $\begin{array}{l}1.31- \\
2.37\end{array}$ & $<0.001$ \\
\hline HTN & 0.76 & $0.60-0.96$ & 0.023 & 1.02 & $\begin{array}{l}0.74- \\
1.40\end{array}$ & 0.902 \\
\hline CAD & 1.51 & $1.03-2.23$ & 0.037 & 1.75 & $\begin{array}{l}1.09- \\
2.80\end{array}$ & 0.021 \\
\hline MELD scores & 1.43 & $1.38-1.49$ & $\begin{array}{l}<.001 \\
0.0\end{array}$ & 1.33 & $\begin{array}{l}1.27- \\
1.39\end{array}$ & $<0.001$ \\
\hline HBV & 0.26 & $0.20-0.32$ & $<.001$ & & & \\
\hline Alcoholic liver cirrhosis & 3.35 & $2.43-4.63$ & $<.001$ & & & \\
\hline
\end{tabular}

$\mathrm{OR}$, odds ratio; $\mathrm{Cl}$, confidence interval; $\mathrm{RDW}$, red cell distribution width; $\mathrm{BMI}$, body mass index; $\mathrm{DM}$, diabetes mellitus; HTN, hypertension; CAD, coronary artery disease; MELD, model for end-stage liver disease; HBV, hepatitis B virus; HCC, hepatocellular carcinoma; NLR, neutrophil to lymphocyte ratio; $\mathrm{PNI}$, prognostic nutritional index.

Values are expressed as means \pm standard deviations, medians (interquartile ranges), or absolute numbers (percentages). 


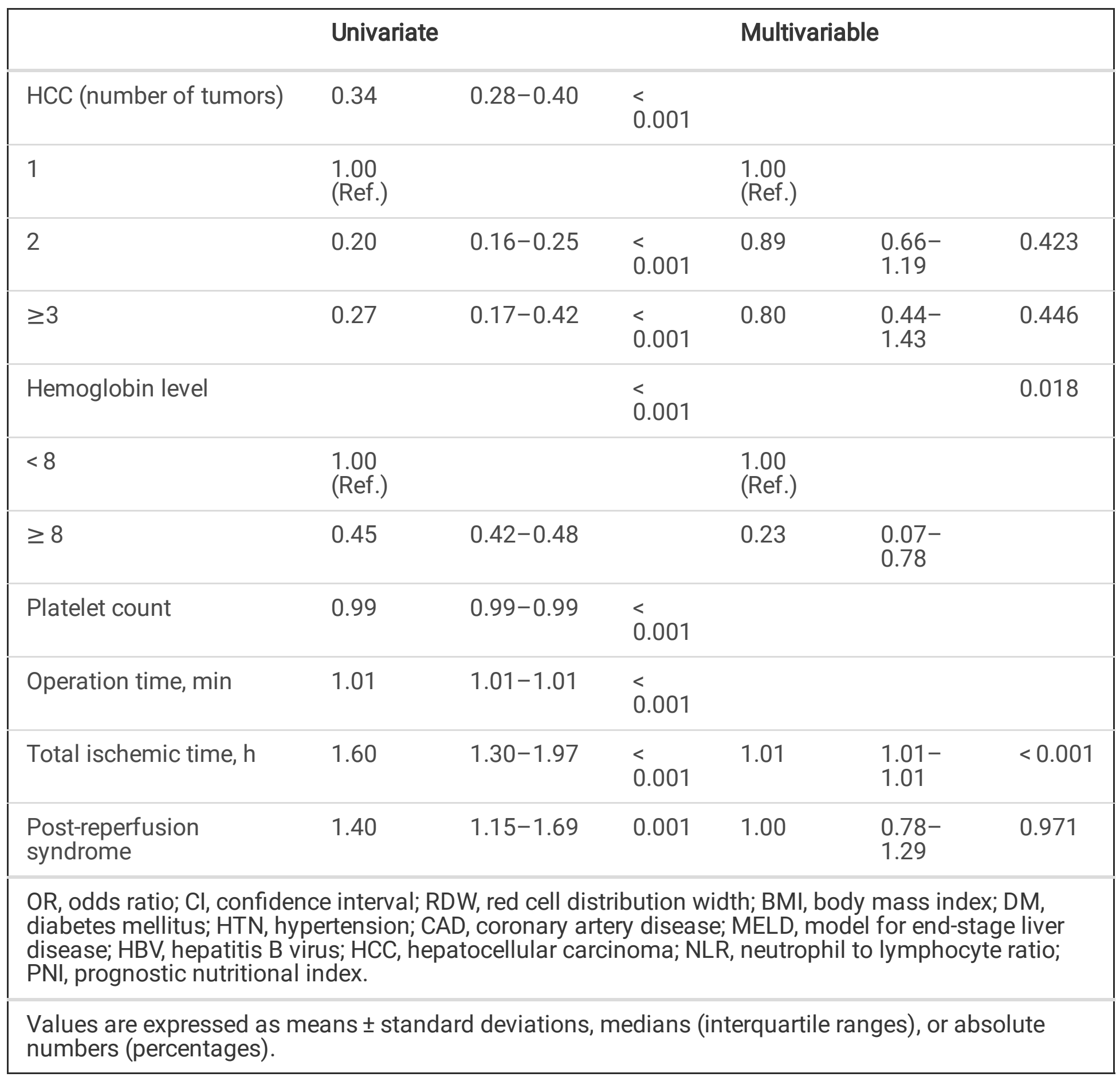

RDW Q4 was significantly associated with postoperative AKI (OR 1.61, 95\% Cl 1.19-2.18, $P=0.002)$ even after adjusting for potentially confounding variables (Table 3 ). 
Table 3

Intraoperative transfusion and postoperative acute kidney injury according to quartiles of preoperative red cell distribution width

\begin{tabular}{|c|c|c|c|c|}
\hline & \multicolumn{2}{|l|}{ Crude } & \multicolumn{2}{|l|}{ Multivariate* } \\
\hline & OR $(95 \% \mathrm{Cl})$ & P-value & OR $(95 \% \mathrm{Cl})$ & $P$-value \\
\hline \multicolumn{5}{|c|}{ Intraoperative transfusion } \\
\hline Quartile $\otimes$ & 1.00 (Ref.) & & 1.00 (Ref.) & \\
\hline Quartile $\otimes$ & $3.98(3.11-5.10)$ & $<0.001$ & $1.93(1.45-2.58)$ & $<0.001$ \\
\hline Quartile $\otimes$ & $12.53(9.09-17.26)$ & $<0.001$ & $4.04(2.79-5.84)$ & $<0.001$ \\
\hline Quartile $\otimes$ & $27.95(18.18-42.98)$ & $<0.001$ & $4.23(2.86-6.93)$ & $<0.001$ \\
\hline \multicolumn{5}{|l|}{ AKI } \\
\hline 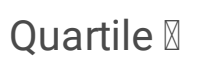 & 1.00 (Ref.) & & 1.00 (Ref.) & \\
\hline 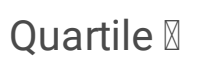 & $1.86(1.48-2.33)$ & $<0.001$ & $1.89(1.49-2.40)$ & $<0.001$ \\
\hline Quartile $\otimes$ & $2.11(1.68-2.64)$ & $<0.001$ & $2.22(1.73-2.86)$ & $<0.001$ \\
\hline 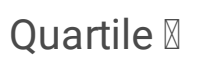 & $1.65(1.32-2.06)$ & $<0.001$ & $1.91(1.44-2.54)$ & $<0.001$ \\
\hline \multicolumn{5}{|c|}{$\begin{array}{l}\text { * Adjusted for age, sex, BMI, DM, HTN, CAD, TNM staging, MELD score, hemoglobin, total ischemic } \\
\text { time, and post-reperfusion syndrome. }\end{array}$} \\
\hline \multicolumn{5}{|c|}{ Study groups according to RDW quartiles: Q1 (<13.9), Q2 (13.9-15.2), Q3 (15.3-17.4), Q4 (> 17.5). } \\
\hline \multicolumn{5}{|c|}{$\begin{array}{l}\text { BMI, body mass index; DM, diabetes mellitus; HTN, hypertension; CAD, coronary artery disease; MELD, } \\
\text { model for end-stage liver disease; RDW, red cell distribution width; AKI, acute kidney injury. }\end{array}$} \\
\hline
\end{tabular}

According to the ROC curve analysis for intraoperative transfusion, the addition of hemoglobin levels to model 1 (MELD scores) improved the area under the curve (AUC: 0.901, $P<0.001$ ) (Table 4 and Fig. 1 ). The addition of RDW to model 2 (MELD scores + hemoglobin) showed no significant improvement in AUC $(P=0.394)$ (Table 4 and Fig. 1). However, an IDI of $0.003(P=0.023)$ indicated that adding RDW to model 2 significantly improved the model's predictive power in terms of a positive difference between increased average sensitivity and any potential increase in average 1-specificity (Table 4). Figure 2 shows the Kaplan-Meier curve according to preoperative RDW quartiles (log-rank test; $P=0.021$ ). One-year survival was significantly different between RDW Q4 and the other quartiles. 
Table 4

Predictive models for intraoperative transfusion

\begin{tabular}{|c|c|c|c|c|c|}
\hline & & AUC difference & $P$-value & IDI & $P$-value \\
\hline \multirow[t]{2}{*}{ Transfusion } & Model 1* vs. 2† & 0.052 & $<0.001$ & 148.000 & $<0.001$ \\
\hline & Model $2+$ vs. $3^{\star \star}$ & 0.001 & 0.394 & 0.003 & 0.023 \\
\hline \multicolumn{6}{|c|}{ * Model 1 = MELD scores } \\
\hline \multicolumn{6}{|c|}{ † Model 2 = MELD scores + hemoglobin level } \\
\hline \multicolumn{6}{|c|}{$\star \star$ Model 3 = MELD scores + hemoglobin level + RDW } \\
\hline \multicolumn{6}{|c|}{$\begin{array}{l}\text { AUC, area under the curve; IDI, integrated discrimination improvement; MELD, model for end-stage live } \\
\text { disease; RDW, red cell distribution width. }\end{array}$} \\
\hline
\end{tabular}

\section{Discussion}

In the present study, we found a significant difference in the incidence of intraoperative transfusion between RDW quartiles (47.3\%, 78.1\%, 91.8\%, and 96.2\% in Q1, Q2, Q3, and Q4, respectively) in patients who underwent LDLT. There was a positive correlation between RDW quartile, massive transfusions, postoperative transfusions, and postoperative AKI. The incidence of intraoperative transfusions and postoperative AKI increased significantly, even after adjusting for potential confounding factors. In addition, IDI analysis showed that RDW, MELD scores, and hemoglobin levels had better predictive power for intraoperative transfusion.

Despite the recent advancements in surgical and anesthetic techniques, intraoperative transfusion and massive bleeding remain major problems in LDLT. Massive bleeding is a predictor of poor surgical outcomes[21] and is linked to increased morbidity and mortality.[22] Blood transfusion is correlated with poor graft survival, infection, and increased complications such as renal failure, bacterial sepsis, and allergic reactions.[23,24] The preoperative identification of factors that can predict the need for blood transfusion and the risk of massive bleeding during LDLT can improve surgical outcomes.

Previous studies have analyzed predictors of bleeding and transfusion during LT.[6, 7, 21, 25-29] Araújo and colleagues reported that preoperative INR, hemoglobin levels, age, and liver pathology were significant predictors of intraoperative blood transfusion in orthotopic liver transplantation (OLT); however, the predictive power of these factors was low.[7] Yuasa and colleagues reported that age, weight, CRP, hematocrit, and total bilirubin were preoperative risk factors for massive blood loss in LDLT. [21] Other studies found a low correlation between bleeding and INR in patients with chronic liver disease. $[29,30]$ The association between the severity of liver disease (Child-Turcotte-Pugh and MELD scores) and perioperative bleeding is controversial.[4,31] Moreover, there is limited information on the risk factors for 
transfusion and bleeding in LDLT. Our study is clinically significant as the first to investigate the link between preoperative RDW and intraoperative transfusion in LDLT patients.

In our study, the incidence of perioperative blood transfusion was significantly associated with preoperative RDW. Additionally, the inclusion of RDW in the model consisting of MELD scores and hemoglobin levels, which are known transfusion risk factors, improved the predictive power for intraoperative blood transfusion, suggesting a strong association between preoperative RDW and perioperative bleeding in LDLT.

In the multivariate logistic regression analysis, preoperative RDW, preoperative hemoglobin level $>8 \mathrm{~g} / \mathrm{dL}$, age, female sex, DM, CAD, MELD scores, and total ischemic time were significantly associated with intraoperative transfusion. Preoperative hemoglobin level has been reported as a risk factor for blood transfusion in many previous studies. $[7,26,28]$ Transfusions occur when hemoglobin levels are low, and in our study, blood transfusions were also based on hemoglobin less than 8; therefore, hemoglobin is one of the strongest triggers of the need for transfusions. Age and MELD scores are risk factors for blood transfusion in LT, which may be due to the patient's systemic condition, comorbidities, and disease severity.[7, 21, 25, 32] A recent study reported that female patients had a higher risk of intraoperative blood transfusion than male patients because of sex differences in preoperative hematocrit and blood volume.[33] DM is related to coagulopathy, microvasculopathy, and a hypercoagulable state,[34, 35] which may increase the risk of intraoperative blood transfusions. CAD is associated with anticoagulants and, therefore, increases the risk of surgical bleeding.[36, 37] However, patients taking anticoagulants were excluded from our analysis. The relationship between CAD and transfusion is thought to be the result of a high standard of transfusion in CAD patients (hemoglobin level $>10 \mathrm{~g} / \mathrm{dL}$ ). Total or warm ischemic time is a risk factor for blood loss in LT patients, which might be associated with postreperfusion syndrome, a possible cause of increased transfusion requirements.[25, 38]

Inflammatory reactions caused by various cytokines can trigger an abnormal clotting system, resulting in hypercoagulation, increasing intraoperative bleeding risk.[39, 40] Decreased liver function associated with nutritional deficiencies and inflammatory status may exacerbate disease severity[41] and increase the risk of bleeding.[42] Recent studies have shown that elevated RDW may be associated with bleeding risk. [14-16] The mechanism by which RDW increases is not yet clear, but it has been reported to be associated with anemia, inflammatory responses and oxidative stress.[43] Increased RDW is a sign of a nutritional deficit, such as a deficiency of iron, folic acid, or vitamins B-12, which may indicate macrocytic anemia and may increase blood transfusion requirements during surgery.[44] The inhibition of erythrocyte maturation by inflammatory cytokines can increase the risk of blood transfusions by causing abnormal erythropoietin function and anisocytosis, which may be associated with anemia and thrombotic conditions.[45]

In our study, patients with higher RDW had poor surgical outcomes, such as prolonged hospital and ICU stay, AKI, increased risk of 1-year graft failure, and lower 1-year and overall survival. Postoperative AKI was significantly associated with RDW, even after adjusting for potentially confounding variables. 
Consistent with previous studies, our results suggest that high RDW is significantly associated with poor surgical prognosis.[10, 12]

This study has some limitations. First, the major limitations of this study are those inherent to its retrospective nature. Thus, the possibility of reporting undocumented factors and a potential bias associated with patient selection and recall existed. However, we tried to reduce the impact of confounding factors by adjusting for variables that could affect the outcome. Second, our patients were admitted to one of the largest LT center in the world, and the LT team had extensive experience, performing more than 300 LDLTs per year since 2010.[46] Therefore, our results may differ from those of studies conducted in other institutions or from multicenter studies and may be difficult to apply to patients undergoing OLT. Third, there are no studies on the accurate validation of preoperative RDW cutoff values that predict surgical outcomes. Therefore, further well-designed research on this topic is needed.

\section{Conclusion}

We found that high preoperative RDW was strongly associated with intraoperative blood transfusion and postoperative AKI in patients undergoing LDLT. These results indicate that preoperative RDW can be a useful predictor of intraoperative blood transfusion and postoperative AKI in LDLT recipients.

\section{Abbreviations}

AKI: acute kidney injury; ALT: alanine transaminase; AST: aspartate transaminase; BMI: body mass index; CAD: coronary artery disease; $\mathrm{Cl}$ : confidence interval; $\mathrm{DM}$ : diabetes mellitus; eGFR: estimated glomerular filtration rate; HBV: hepatitis B virus; HCC: hepatocellular carcinoma; HCV: hepatitis C virus; $\mathrm{HTN}$ : hypertension; ICU: intensive care unit; IDI: integrated discrimination improvement; INR: international normalized ratio; LDLT: living donor liver transplantation; MBP: mean blood pressure; MELD: model for end-stage liver disease; NLR: neutrophil-lymphocyte ratio; OR: odds ratio; PLR: platelet-lymphocyte ratio; PNI: prognostic nutritional index; PRBC: packed red blood cell; RDW: red cell distribution width; ROC: receiver operating characteristic; sCr: serum creatinine; WBC: white blood cell.

\section{Declarations}

\section{Ethics approval and consent to participate}

The need for informed consent from individual patients was waived owing to the retrospective nature of the study.

\section{Consent for publication}

Not applicable 
Availability of data and materials

The dataset used and/or analyzed during the current study is available from the corresponding author on reasonable request.

\section{Competing interests}

The authors declare that they have no conflicts of interest.

\section{Funding}

This work was supported by a grant of the Korea Health Technology R\&D Project (Grant No. HI18C2383) through the Korea Health Industry Development Institute affiliated to the Ministry of Health and Welfare of the Republic of Korea.

\section{Authors' contribution}

JHS, JGS and GSH conceived and designed the study; JHS, HMK, IGJ, SHK and JGS were involved in data acquisition; JHS, HMK, IGJ, BK, SK and GSH were involved in analysis and/or interpretation of data; JHS drafted the manuscript; JGS revised the manuscript critically for important intellectual content. All authors gave approval for the final manuscript.

\section{Acknowledgements}

None.

\section{References}

1. Starzl TE, Demetris AJ, Van Thiel D. Liver transplantation (1). N Engl J Med. 1989;321:1014-22.

2. Lewis JH, Bontempo FA, Cornell F, Kiss JE, Larson P, Ragni MV, et al. Blood use in liver transplantation. Transfusion. 1987;27:222-5.

3. Frasco PE, Poterack KA, Hentz JG, Mulligan DC. A comparison of transfusion requirements between living donation and cadaveric donation liver transplantation: relationship to model of end-stage liver disease score and baseline coagulation status. Anesth Analg. 2005;101:30-7, table of contents.

4. Massicotte L, Denault AY, Beaulieu D, Thibeault L, Hevesi Z, Nozza A, et al. Transfusion rate for 500 consecutive liver transplantations: experience of one liver transplantation center. Transplantation. 2012;93:1276-81.

5. Hannaman MJ, Hevesi ZG. Anesthesia care for liver transplantation. Transplant Rev (Orlando). 2011;25:36-43.

6. Steib A, Freys G, Lehmann C, Meyer C, Mahoudeau G. Intraoperative blood losses and transfusion requirements during adult liver transplantation remain difficult to predict. Can $\mathrm{J}$ Anaesth. 2001;48:1075-9. 
7. Araújo T, Cordeiro A, Proença P, Perdigoto R, Martins A, Barroso E. Predictive variables affecting transfusion requirements in orthotopic liver transplantation. Transplant Proc. 2010;42:1758-9.

8. Salvagno GL, Sanchis-Gomar F, Picanza A, Lippi G. Red blood cell distribution width: A simple parameter with multiple clinical applications. Crit Rev Clin Lab Sci. 2015;52:86-105.

9. Parizadeh SM, Jafarzadeh-Esfehani R, Bahreyni A, Ghandehari M, Shafiee M, Rahmani F, et al. The diagnostic and prognostic value of red cell distribution width in cardiovascular disease; current status and prospective. Biofactors. 2019;45:507-16.

10. Wang PF, Song SY, Guo H, Wang TJ, Liu N, Yan CX. Prognostic role of pretreatment red blood cell distribution width in patients with cancer: A meta-analysis of 49 studies. J Cancer. 2019;10:4305-17.

11. Pedrazzani C, Tripepi M, Turri G, Fernandes E, Scotton G, Conci S, et al. Prognostic value of red cell distribution width (RDW) in colorectal cancer. Results from a single-center cohort on 591 patients. Sci Rep. 2020;10:1072.

12. Jia L, Cui S, Yang J, Jia Q, Hao L, Jia R, et al. Red blood cell distribution width predicts long-term mortality in critically ill patients with acute kidney injury: a retrospective database study. Sci Rep. 2020;10:4563.

13. Patel R, English L, Liu WK, Tree AC, Ayres B, Watkin N, et al. Red cell differential width (RDW) as a predictor of survival outcomes with palliative and adjuvant chemotherapy for metastatic penile cancer. Int Urol Nephrol. 2020;52:2301-6.

14. Altintas O, Duruyen H, Baran G, Baran O, Katar S, Antar V, et al. The Relationship of Hematoma Growth to Red Blood Cell Distribution Width in Patients with Hypertensive Intracerebral Hemorrhage. Turk Neurosurg. 2017;27:368-73.

15. Lee KR, Park SO, Kim SY, Hong DY, Kim JW, Baek KJ, et al. Red cell distribution width as a novel marker for predicting high-risk from upper gastro-intestinal bleeding patients. PLoS One. 2017;12:e0187158.

16. Fatemi O, Torguson R, Chen F, Ahmad S, Badr S, Satler LF, et al. Red cell distribution width as a bleeding predictor after percutaneous coronary intervention. Am Heart J. 2013;166:104-9.

17. Kim YK, Shin WJ, Song JG, Jun IG, Kim HY, Seong SH, et al. Effect of right ventricular dysfunction on dynamic preload indices to predict a decrease in cardiac output after inferior vena cava clamping during liver transplantation. Transplant Proc. 2010;42:2585-9.

18. Onodera T, Goseki N, Kosaki G. [Prognostic nutritional index in gastrointestinal surgery of malnourished cancer patients]. Nihon Geka Gakkai Zasshi. 1984;85:1001-5.

19. Pham HP, Shaz BH. Update on massive transfusion. Br J Anaesth. 2013;111 Suppl 1:i71-82.

20. Luo X, Jiang L, Du B, Wen $Y$, Wang M, Xi X. A comparison of different diagnostic criteria of acute kidney injury in critically ill patients. Crit Care. 2014;18:R144.

21. Yuasa T, Niwa N, Kimura S, Tsuji H, Yurugi K, Egawa H, et al. Intraoperative blood loss during living donor liver transplantation: an analysis of 635 recipients at a single center. Transfusion. 2005;45:879-84. 
22. Cleland S, Corredor C, Ye JJ, Srinivas C, McCluskey SA. Massive haemorrhage in liver transplantation: Consequences, prediction and management. World journal of transplantation. 2016;6:291-305.

23. Massicotte L, Sassine MP, Lenis S, Seal RF, Roy A. Survival rate changes with transfusion of blood products during liver transplantation. Can J Anaesth. 2005;52:148-55.

24. Boin IF, Leonardi MI, Luzo AC, Cardoso AR, Caruy CA, Leonardi LS. Intraoperative massive transfusion decreases survival after liver transplantation. Transplant Proc. 2008;40:789-91.

25. Eghbal MH, Samadi K, Khosravi MB, Sahmeddini MA, Ghaffaripoor S, Ghorbani M, et al. The Impact of Preoperative Variables on Intraoperative Blood Loss and Transfusion Requirements During Orthotopic Liver Transplant. Exp Clin Transplant. 2019;17:507-12.

26. Pandey CK, Singh A, Kajal K, Dhankhar M, Tandon M, Pandey VK, et al. Intraoperative blood loss in orthotopic liver transplantation: The predictive factors. World J Gastrointest Surg. 2015;7:86-93.

27. Yoon JU, Byeon GJ, Park JY, Yoon SH, Ryu JH, Ri HS. Bloodless living donor liver transplantation: Risk factors, outcomes, and diagnostic predictors. Medicine (Baltimore). 2018;97:e13581.

28. Choi SS, Cho SS, Kim SH, Jun IG, Hwang GS, Kim YK. Factors associated with blood transfusion in donor hepatectomy: results from 2344 donors at a large single center. Transplantation. 2013;96:1000-7.

29. Caldwell SH, Hoffman M, Lisman T, Macik BG, Northup PG, Reddy KR, et al. Coagulation disorders and hemostasis in liver disease: pathophysiology and critical assessment of current management. Hepatology. 2006;44:1039-46.

30. Massicotte L, Beaulieu D, Thibeault L, Roy JD, Marleau D, Lapointe R, et al. Coagulation defects do not predict blood product requirements during liver transplantation. Transplantation. 2008;85:956-62.

31. McCluskey SA, Karkouti K, Wijeysundera DN, Kakizawa K, Ghannam M, Hamdy A, et al. Derivation of a risk index for the prediction of massive blood transfusion in liver transplantation. Liver Transpl. 2006;12:1584-93.

32. Alonso IJ, Maestro OC, Gómez NF, Mateos RS, Quinto AM, Molero FC, et al. Mind the model for endstage liver disease: model for end-stage liver disease score as an indicator of hemoderivate transfusion and survival in liver transplantation. Transplant Proc. 2015;47:97-9.

33. Stammers AH, Tesdahl EA, Mongero LB, Stasko A. Gender and intraoperative blood transfusion: analysis of 54,122 non-reoperative coronary revascularization procedures. Perfusion. 2019;34:23645.

34. Alzahrani SH, Ajjan RA. Coagulation and fibrinolysis in diabetes. Diab Vasc Dis Res. 2010;7:260-73.

35. Sobel BE, Schneider DJ. Platelet function, coagulopathy, and impaired fibrinolysis in diabetes. Cardiol Clin. 2004;22:511-26.

36. Hedge J, Balajibabu PR, Sivaraman T. The patient with ischaemic heart disease undergoing non cardiac surgery. Indian J Anaesth. 2017;61:705-11. 
37. Rodriguez A, Guilera N, Mases A, Sierra P, Oliva JC, Colilles C. Management of antiplatelet therapy in patients with coronary stents undergoing noncardiac surgery: association with adverse events. $\mathrm{Br} J$ Anaesth. 2018;120:67-76.

38. De Santis GC, Brunetta DM, Nardo M, Oliveira LC, Souza FF, Cagnolati D, et al. Preoperative variables associated with transfusion requirements in orthotopic liver transplantation. Transfus Apher Sci. 2014;50:99-105.

39. Krzanicki D, Sugavanam A, Mallett S. Intraoperative hypercoagulability during liver transplantation as demonstrated by thromboelastography. Liver Transpl. 2013;19:852-61.

40. Li MX, Zhang XF, Liu ZW, Lv Y. Risk factors and clinical characteristics of portal vein thrombosis after splenectomy in patients with liver cirrhosis. Hepatobiliary Pancreat Dis Int. 2013;12:512-9.

41. Bothe A, Jr., Steele G, Jr. Is there a role for perioperative nutritional support in liver resection? HPB Surg. 1997;10:177-9.

42. Ponziani FR, Zocco MA, Campanale C, Rinninella E, Tortora A, Di Maurizio L, et al. Portal vein thrombosis: insight into physiopathology, diagnosis, and treatment. World J Gastroenterol. 2010;16:143-55.

43. Pierce CN, Larson DF. Inflammatory cytokine inhibition of erythropoiesis in patients implanted with a mechanical circulatory assist device. Perfusion. 2005;20:83-90.

44. Sultana GS, Haque SA, Sultana T, Ahmed AN. Value of red cell distribution width (RDW) and RBC indices in the detection of iron deficiency anemia. Mymensingh Med J. 2013;22:370-6.

45. Byrnes JR, Wolberg AS. Red blood cells in thrombosis. Blood. 2017;130:1795-9.

46. Moon DB, Lee SG, Hwang S, Kim KH, Ahn CS, Ha TY, et al. More than 300 consecutive living donor liver transplants a year at a single center. Transplant Proc. 2013;45:1942-7.

\section{Figures}




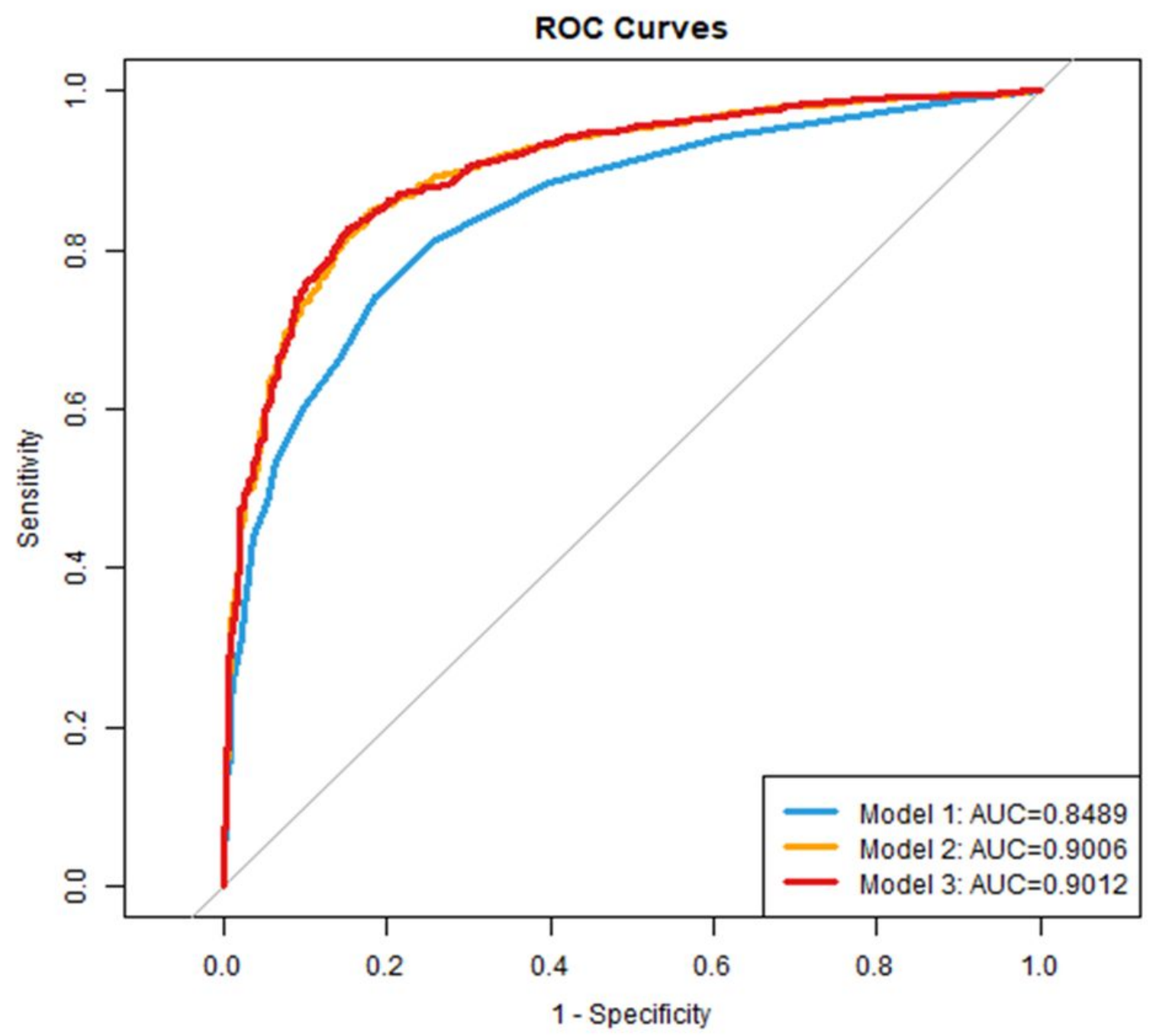

Figure 1

Receiver operating characteristic analyses of predictive models for intraoperative transfusion in patients who underwent living donor liver transplantation. Model $1=$ MELD Model $2=$ MELD + hemoglobin levels Model 3 = MELD + hemoglobin levels + RDW MELD, model for end-stage liver disease; RDW, red cell distribution width 


\section{1-year survival}

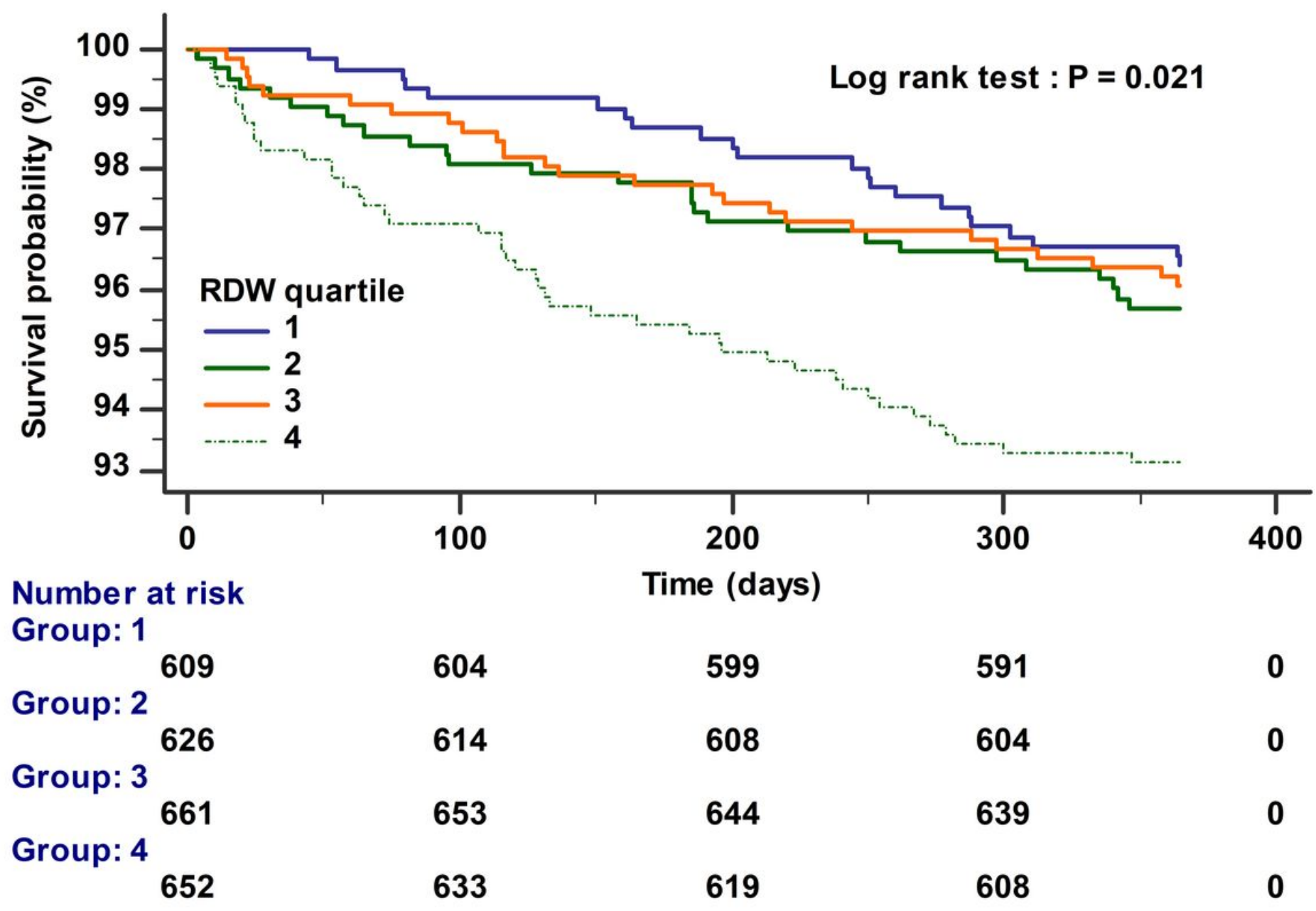

Figure 2

Kaplan-Meier survival curve for 1-year survival according to quartiles of preoperative red cell distribution width. The survival rate was significantly poor in patients with preoperative RDW level of quartile 4 than in those with preoperative RDW level of quartile 1, 2, and 3 (log-rank test; $p=0.021$ ).

\section{Supplementary Files}

This is a list of supplementary files associated with this preprint. Click to download.

- VisualAbstractcriticalcare.jpg 